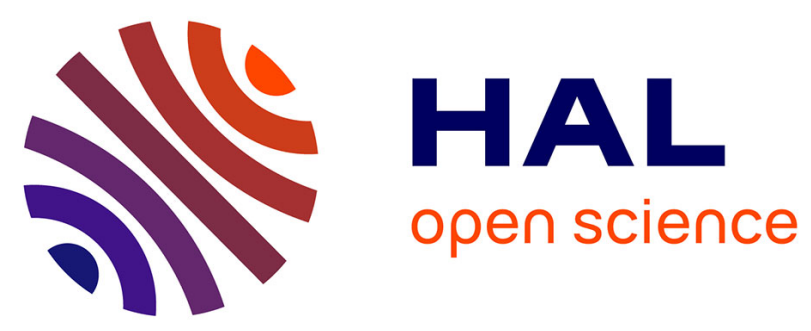

\title{
Characterisation of cubic oak specimens from the Vasa ship and recent wood by means of quasi-static loading and resonance ultrasound spectroscopy (RUS)
}

Alexey Vorobyev, Olivier Arnould, Didier Laux, Roberto Longo, Nico van Dijk, E. Kristofer Gamstedt

\section{To cite this version:}

Alexey Vorobyev, Olivier Arnould, Didier Laux, Roberto Longo, Nico van Dijk, et al.. Characterisation of cubic oak specimens from the Vasa ship and recent wood by means of quasi-static loading and resonance ultrasound spectroscopy (RUS). Holzforschung, 2015, 10.1515/hf-2015-0073 . hal01219461

\section{HAL Id: hal-01219461 \\ https://hal.science/hal-01219461}

Submitted on 22 Oct 2015

HAL is a multi-disciplinary open access archive for the deposit and dissemination of scientific research documents, whether they are published or not. The documents may come from teaching and research institutions in France or abroad, or from public or private research centers.
L'archive ouverte pluridisciplinaire HAL, est destinée au dépôt et à la diffusion de documents scientifiques de niveau recherche, publiés ou non, émanant des établissements d'enseignement et de recherche français ou étrangers, des laboratoires publics ou privés. 
Alexey Vorobyev*, Olivier Arnould, Didier Laux, Roberto Longo, Nico P. van Dijk

and E. Kristofer Gamstedt

\section{Characterisation of cubic oak specimens from the Vasa ship and recent wood by means of quasi-static loading and resonance ultrasound spectroscopy (RUS)}

DOI 10.1515/hf-2015-0073

Received March 17, 2015; accepted August 10, 2015; previously published online $x x$

\begin{abstract}
The cylindrical orthotropy, inherent timedependency response, and variation between and within samples make the stiffness characterisation of wood more challenging than most other structural materials. The purpose of the present study is to compare static loading with resonant ultrasound spectroscopy (RUS) and to investigate how to combine the advantages of each of these two methods to improve the estimation of the full set of elastic parameters of a unique sample. The behavior of wood as an orthotropic mechanical material was quantified by elastic engineering parameters, i.e. Poisson's ratios and Young's and shear moduli. Recent and waterlogged archaeological oak impregnated with polyethylene glycol (PEG) from the Vasa warship built in 1628 was in focus. The experimental results were compared, and the difference between RUS and static loading was studied. This study contributes additional information on the influence of PEG and degradation on the elastic engineering parameters of wood. Finally, the shear moduli and Poisson's ratios were experimentally determined for Vasa archaeological oak for the first time.
\end{abstract}

*Corresponding author: Alexey Vorobyev, Division of Applied Mechanics, Ångström Laboratory, Uppsala University, Box 534, SE-751 21 Uppsala, Sweden, e-mail: alexey.vorobyev@me.com alexey.vorobyev@angstrom.uu.se

Olivier Arnould: Université de Montpellier, LMGC, CNRS UMR 5508, F-34095 Montpellier, France

Didier Laux: Université de Montpellier, IES, CNRS UMR 5214, F-34095, Montpellier, France

Roberto Longo: ESEO Group - GSII, 49107 Angers, France; and L'UNAM University, LAUM, CNRS UMR 6613, 72085 Le Mans, France Nico P. van Dijk and E. Kristofer Gamstedt: Division of Applied Mechanics, Ångström Laboratory, Uppsala University, Box 534, SE-751 21 Uppsala, Sweden
Keywords: archaeological wood, compression test, cubic samples, elastic constants, oak wood, quasi-static loading, resonant ultrasound spectroscopy (RUS), Vasa ship

\section{Introduction}

The modulus of elasticity (MOE) is a good parameter for the grading of wood. It is obtained from bending tests on large wooden beams. In a more careful design of wood structure, the full orthotropic set of elastic parameters is needed (Tsoumis 1991; Smith et al. 2003), e.g. for the finite-element modelling of wood joints that are locally subjected to a triaxial stress state. The most straightforward way of obtaining the elastic properties is through quasi-static loading, where the load is applied at a very slow rate to avoid any dynamic effects (Kollmann 1968). The absolute values obtained are then close to the most common conditions prevalent in the first design step. For the Young's moduli, tensile testing of "dog-bone" specimens in the longitudinal (L), tangential $(\mathrm{T})$, and radial $(\mathrm{R})$ directions is preferred, because a uniaxial and homogeneous stress state is achieved in the gauge length (Ozyhar et al. 2013), but sample matching is not guaranteed. For precious wood material, e.g. archaeological wood, it is often better to investigate cubic samples loaded in compression, as a single sample can be loaded elastically by applying load consecutively in all three directions (Ljungdahl and Berglund 2007), if loading is restricted to the reversible elastic region. Thus, material can be saved, and measurements are made on the same sample. A disadvantage of this approach is that the evaluation of data obtained from pure compressive stresses is more complicated due to the inhomogeneous distribution of the applied compressive force due to geometrical imperfections in the test specimen (Toftegaard 1999).

In addition, barrelling formation in compressive loading, due to the more or less restrained contact slip on the platen area, makes it difficult to assess the transverse 
deformations without finite-element modelling. The Poisson's ratios are thus more difficult to deduce directly from this kind of loading. Strain field measurements by digital image correlation (DIC) are often required to estimate the stiffness parameters accurately (Dahl and Malo 2009; Majano-Majano et al. 2012; Xavier et al. 2012; Ozyhar et al. 2013). In addition, the cylindrical orthotropy and curvature of the annual rings make the stress field even more non-uniform for large specimens. For too small specimens, the tested volume may not be representative, leading to a large scatter and uncertainty in the measured stiffness. Nevertheless, compression testing of wooden cubes is the reasonable choice of test method if the amount of available material is limited. Shear testing is generally more complex. However, the same cubic samples can also be used to characterise the quasi-static shear moduli in a test rig designed to induce a state close to pure and homogeneous shear over a relatively large volume in the center of the specimen (Hassel et al. 2009). Again, the cubic shape allows for testing in all three material directions if the loading is restricted to the reversible elastic region.

Ultrasound (US) transmission measurements (Bucur 2006) are a fast and cheap alternative for estimating the diagonal terms of the elastic compliance or stiffness tensor, but very high frequencies are needed (usually in the order of some $\mathrm{MHz}$ ) compared to quasi-static tests. The accurate determination of the Poisson's ratios using this technique is not trivial (Gonçalves et al. 2011, 2014; Vázquez et al. 2015). Moreover, anatomic variation can limit the accuracy, because wood cells, vessels, rays, latewood/earlywood (LW/EW) layers, etc., can be comparable in size with the US wavelength. Finally, the elastic quantity (i.e. elastic modulus or compliance) measured by means of longitudinal US waves is still not clear for geometry such as a cube (Gonçalves et al. 2011; Rakotovololonalimanana et al. 2015; Vázquez et al. 2015).

An alternative, quite recent method in wood science, is resonant ultrasound spectroscopy (RUS) that relies on the mechanical resonances of samples that have simple geometrical shapes (typically cubic, cylindrical, or spherical) to measure the elastic tensor (Migliori et al. 2001). The basic idea behind this method is first to measure as much as possible the natural resonant frequencies and associated mode shapes of the wooden sample. The possibility of quickly measuring mechanical properties without any direct contact is attractive. The application of RUS to wood was first proposed by Schubert et al. (2006) for the determination of one shear modulus. Longo et al. $(2012,2014)$ showed that all the components of the elastic tensor of wood material may be measured on a single specimen. Although the method is performed under dynamic conditions, all the necessary stiffness parameters may be obtained for triaxial stress analysis. RUS gives the dynamic stiffness at intermediate frequencies (i.e. typically around some hundreds of $\mathrm{kHz}$ ), so the strain rate is more than 5 orders of magnitude higher during RUS experiments than in the static compression test, but it has the already mentioned advantage of theoretically yielding the full elastic tensor and of being faster than a compression test. For RUS, tens of minutes are usually needed for only two sample orientations compared to at least nine different experiments in a compression test, which thus required a full working day per sample. The RUS approach on wood is not, however, sufficiently robust up to now, especially for the determination of the Poisson's ratios and the longitudinal Young's modulus (Longo et al. 2014).

The basic idea presented in the present paper is that the advantages of the two methods can be combined. The absolute values of the axial Young's modulus and the Poisson's ratios are first determined from static compression testing, and the relative values of the remaining elastic parameters are then derived from RUS. The hypothesis is that the combination of the data sets would permit a robust inverse identification of the full elastic tensor with a single wood sample. To test this hypothesis, recent oak (Quercus robur L.) samples were investigated together with precious archaeological wood material from the $17^{\text {th }}$ century Vasa warship (Cederlund and Hocker 2006). The elastic, ultimate, and creep properties of Vasa oak are required for stress analysis to design a better support structure for the ship. The waterlogged Vasa oak has been impregnated with polyethylene glycol (PEG) to prevent cracking and deformation during drying. Both the aging (Bjurhager et al. 2012) and the PEG impregnation (Ljungdahl and Berglund 2007; Bjurhager et al. 2010) have affected the mechanical properties. In this work, the static and dynamic elastic properties of recent and Vasa oak were ascertained by means of a quasi-static compression test and RUS, and the diagonal terms of the elastic stiffness tensor were confirmed by US transmission measurements.

\section{Materials and methods}

Specimens: A compromise must be made between the limited availability of material (small samples preferable) and specimen representativeness (large samples preferable compared to the ring width). For the RUS and the static tests, a total of four cubic samples in total were taken from a block of Vasa material and from a recent oak log as reference. The Vasa material (reference number 65742) is from the keel structure of the ship where holes were made for ventilation. Samples with annual rings having a large radius of curvature were chosen to 
ensure relatively uniform material directions within the cube. All faces of the cubes were machined to give edges of $25 \pm 0.05 \mathrm{~mm}$. The size of the sample was chosen to be larger than the sample heterogeneity, i.e. an average ring width of around $1.5-2 \mathrm{~mm}$. The size of a sample strongly influences the frequencies at which its normal modes occur (Migliori et al. 2001; Zadler et al. 2004), and most of them, especially the lowest ones, must fall within the measurement range and be compatible with the excitation capabilities of our RUS device (frequencies up to a hundred kHz). Finally, the sample side length must be greater than the higher US wavelength in the US transmission measurements (Bucur 2006; Vázquez et al. 2015), i.e. around $5 \mathrm{~mm}$ for the 1-MHz longitudinal transducer in the $\mathrm{L}$ direction. Care was taken to machine the samples with the surfaces oriented as closely as possible along the longitudinal (L), radial (R), and tangential (T) anatomical axes, as shown in Figure 1a. However, in some cases, the misalignment can locally be up to $20^{\circ}$ in the RT plane (Figure 1a). Before testing, the specimens were conditioned in a desiccator at $22^{\circ} \mathrm{C}$ and $55 \% \mathrm{RH}$ until equilibrium was achieved. The specimens were weighed before and after each experiment to ensure that they had similar moisture contents (MCs) for all the measurements.

Static testing: The static tests were performed on a Shimadzu Autograph AG-X universal testing machine (Tokyo, Japan) with a 10-kN load cell. Initial tests were conducted on dummy wood specimens in order to find the approximate elastic loading range for all specimen orientations. The compression test was repeated twice on each specimen in each L, R, and T direction (Figure 1a). Both loading and unloading cycles were recorded as shown in the example in Figure 2. The Young's modulus was determined from the unloading part, as slight surface irregularities of the sample or loading platen are likely to induce local plasticity during the loading part and an increase in the contact area between the sample and the platen. During the unloading, however, these effects should be stabilised, and the contact area should be close to the whole surface area reached at the maximum load in the elastic region. Conventionally, strains are measured by a built-in measurement system or strain gauges. Nowadays, full field strain measurement techniques such as digital speckle photography (Sjödahl and Synnergren 1999) or three-dimensional/stereo-DIC (Majano-Majano et al. 2012) are increasingly common. Strain measurement by DIC has shown good performance in comparison with traditional strain gauges (Huang et al. 2010; Xavier et al. 2012). In our case, full-field displacements were observed with a DIC equipment GOM Aramis stereo system 5M (Braunschweig, Germany). The distance from the measured object was $300 \mathrm{~mm}$. Each surface of the specimen was spray painted with speckles for better contrast. The applied force values were continuously recorded by the DIC system during testing, and these values were stored together with the sampled images. The displacement rate was $0.5 \mathrm{~mm} \mathrm{~min}^{-1}$. a
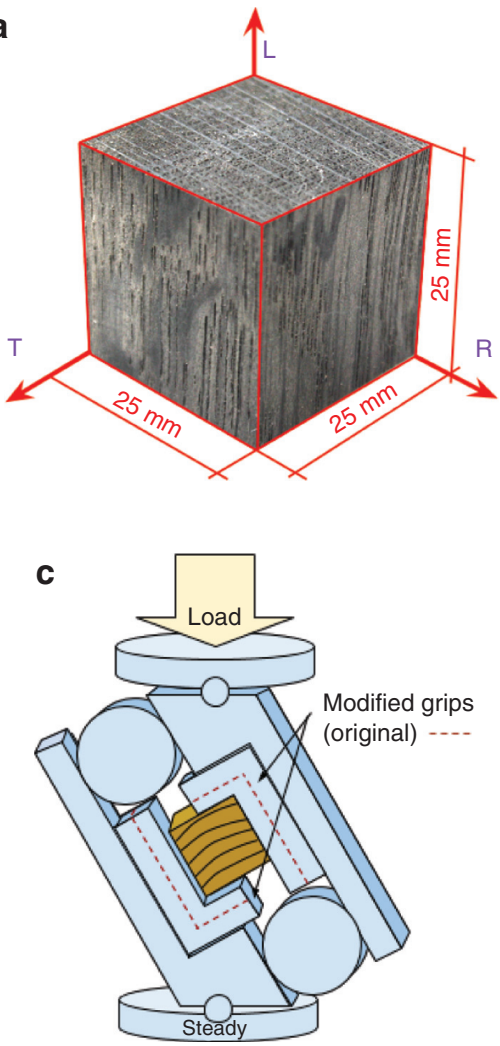

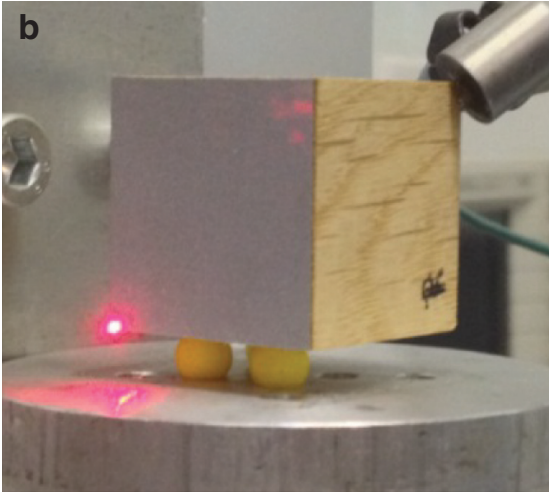

d

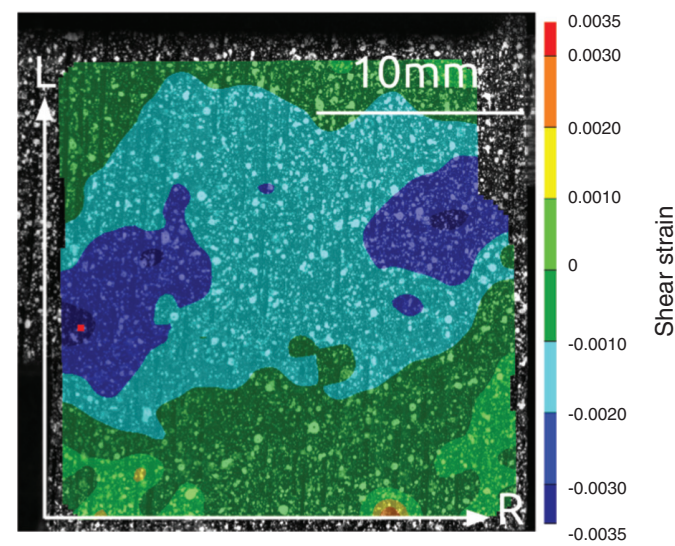

Figure 1: (a) Cubic Vasa oak sample for mechanical testing: identification of anatomical directions and growth ring misalignment in the RT plane. The cubic specimen edge is $25 \mathrm{~mm}$. (b) Recent oak sample in the RUS device during the measurement: reflective tape on the measured plane, laser velocimeter red spot, and piezoelectric ultrasonic transducer on the specimen corner. (c) Modified SCA for shear testing after Hassel et al. (2009). (d) Shear strain field $\varepsilon_{x y}$ obtained by DIC of Vasa oak in the RL plane. With the applied load of $901 \mathrm{~N}$, a shear modulus of $0.62 \mathrm{GPa}$ was obtained for this strain field. 


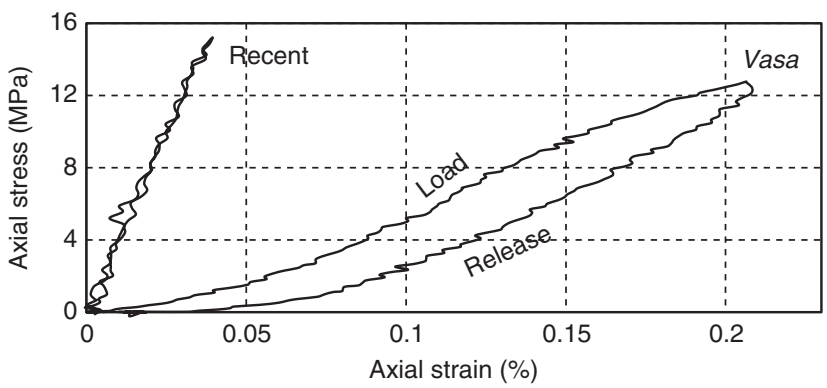

Figure 2: Axial compression stress-strain plot with load-release curves. The PEG-treated Vasa oak shows a high degree of viscoelasticity (i.e. hysteresis loop in the loading/unloading curve).

The ambient conditions were $23^{\circ} \mathrm{C}$ and $51 \%$ RH. All tests were performed without delay after the samples have been removed from the conditioning chamber, so that the mc would essentially be the same after conditioning. The measurement sampling frequency was $1 \mathrm{~Hz}$. Every frame recorded by the DIC system was compared with the undeformed state in order to calculate the displacement and strain fields. The area of interest (a centered square of $400 \mathrm{~mm}^{2}$ ), where a uniform deformation field was found, was subjected to image analysis. The acquired strain fields were smoothed by averaging each data pixel with the surrounding pixels in a $3 \times 3$ array. The applied stress was plotted against the strain determined by DIC in the loading direction (Figure 2).

Poisson's ratios $v_{\mathrm{ij}}$ for all the corresponding orthotropic planes were calculated as a negative ratio between the average transverse $\varepsilon_{i j}$ (passive) and normal $\varepsilon_{i i}$ (compressive and active) strains. The average transverse and normal strains measured together with normal strains were acquired with the DIC technique.

The shear moduli $G_{R L}, G_{T L}$, and $G_{R T}$ were measured on a single sample, oriented for each corresponding symmetry planes RT, LT, and LR, in the test rig called single cube apparatus (SCA) proposed by Hassel et al. (2009). The SCA gives rise to an almost pure shear strain state in the center of the specimen that requires the analysis of full field strain. In the original study, the device was proposed for cubes with quadratic cross-sections of $40 \times 40 \mathrm{~mm}^{2}$. Because of the limitation in size of archaeological samples and the requirements for the RUS measurements, the grips were modified for smaller cross-sections of $25 \times 25$ $\mathrm{mm}^{2}$, as shown in Figure 1c. The shear testing rig was placed in the same Shimadzu mechanical testing machine, where the displacement rate was $1 \mathrm{~mm} \mathrm{~min}^{-1}$. Preliminary tests were carried out on recent oak dummies to determine the approximate elastic regime and the onset of yielding for the different shear planes. The displacement fields were registered with DIC, where images were taken at a frequency of $1 \mathrm{~Hz}$. From the displacement fields, the strain fields for the normal strains $\varepsilon_{y y}$ and $\varepsilon_{x x}$ and for shear strain $\varepsilon_{x y}$ were derived. An example of the strain field is shown in Figure 1d for $\varepsilon_{x y}$ shear strain in the RL plane. Hassel et al. (2009) described the procedure for choosing a suitable region for determining shear strains to estimate the shear modulus.

RUS: The method has been described by Longo et al. (2012, 2014). The specimen was excited by a piezoelectric ultrasonic transducer Panametrics V110RM (Olympus, Tokyo, Japan) and a specific multi-harmonic signal generated by an arbitrary wave generator Agilent 33220A (Santa Rosa, CA, USA), which was connected to the specimen corner with a honey droplet as shown in Figure $1 \mathrm{~b}$. This multi-harmonic signal makes possible the fast and simultaneous measurement of all the measured frequencies without any sweep. A chosen face of the excited cube, covered by a reflective tape, was scanned point by point by means of a laser velocimeter Polytec OFV-505 (Waldbronn, Germany) equipped with two-step motors Physik Instrumente (Karlsruhe, Germany) and driven by National Instruments (Austin, TX, USA) Labview software, and the mode shape at each resonance frequency was obtained. The global frequency range is typically between 5 and $150 \mathrm{kHz}$, depending on the laser acquisition card measurement range and the excitation capabilities. Generally, $40 \times 40$ point maps were recorded. In order to increase the signal-to-noise ratio, around 150 frequency signals were acquired and averaged at each measurement point. As an example, one of the frequency spectra is presented in Figure 3.

For each frequency found in the multi-harmonic excitation signal, the amplitude of the Fourier transform and the phase shift were computed to yield the out-of-plane amplitude of the displacements for each measured point on the cube surface. For a given resonance frequency, its spatial evolution represents exactly the mode shape. The first six measured and identified mode shapes for the recent oak material are presented in Figure 4 as an example.

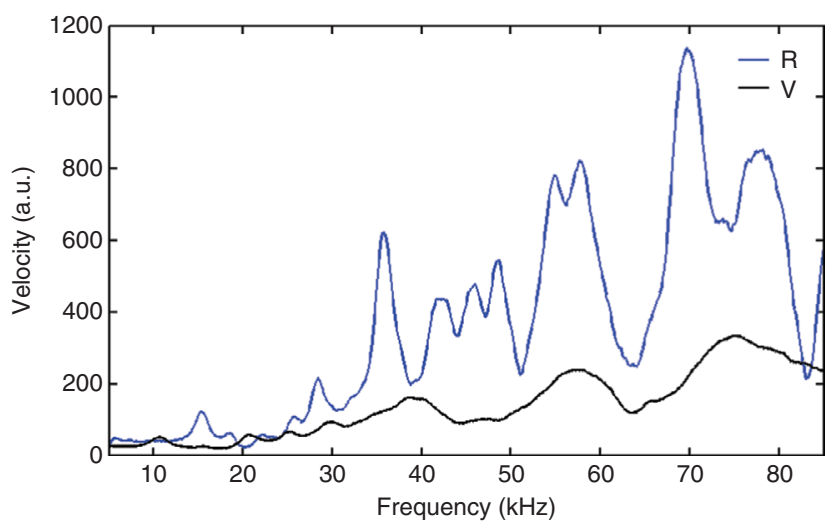

Figure 3: RUS frequency spectra for Vasa $(\mathrm{V})$ and recent (R) oak sample.

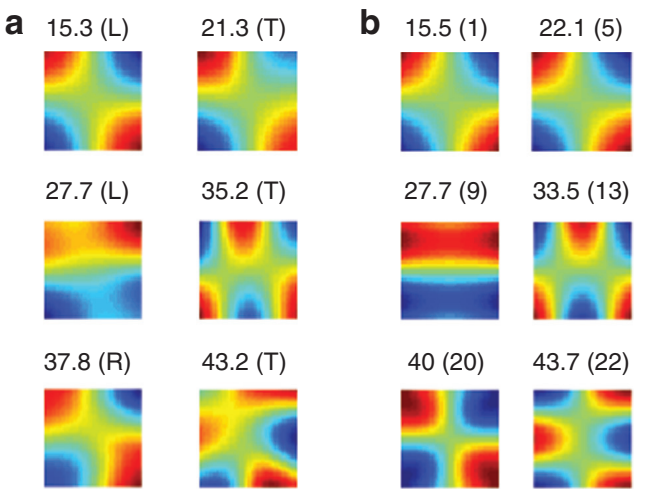

Figure 4: Mode shapes of recent oak sample and their corresponding frequency (kHz). (a) First six measured mode shapes, where the R, T, and $L$ orthotropic directions (in brackets) correspond to the normal to the measured plane (e.g. $R$ is normal to the TL plane). (b) Corresponding identified mode shapes, with the mode numbers within all the theoretical computed ones (in brackets), see Longo et al. (2014). 
The elastic constants were identified using a home-made Matlab (Mathworks, Natick, MA, USA) procedure for the comparison of measured and computed resonance frequencies and mode shapes (Longo et al. 2014). The inverse problem is solved iteratively and needs initial guesses for the elastic constants that must be relatively close to the sought solution. The initial elastic stiffness tensor introduced here for the reference samples was obtained from Guitard's regression from the sample density (Guitard and El Amri 1987). This was not possible for the Vasa material due to the presence of PEG. As no reference shear moduli values exist for the Vasa material, the US transmission method was applied to obtain the initial value of the diagonal terms of the elastic stiffness tensor. The off-diagonal terms were computed so that the Poisson's ratios matched the data obtained by the Guitard regression.

US transmission measurements: This method makes it possible to estimate the diagonal terms of the elastic stiffness tensor considering the geometry of a sample with a small aspect ratio such as a cube (Bucur 2006; Rakotovololonalimanana et al. 2015). Two types of US waves were used: longitudinal waves (Panametrics V103 transducers, Olympus, Tokyo, Japan) and shear waves (Panametrics V151 transducers, Olympus, Tokyo, Japan). For each shear modulus, two velocities were measured because the shear waves are polarised, and they were always almost equal. To improve the $\mathrm{S} / \mathrm{N}$ ratio, 50 transmitted signals captured by the receiving transducer were acquired 100 times by means of a digital oscilloscope LeCroy WaveJet 334 (Teledyne, New York, NY, USA), and the data were averaged. From the mean thickness of the sample in the wave propagation direction and the signal transmission times (obtained with the two transducers directly in contact), the US velocity was deduced. From the sample density and the velocity of longitudinal and transversal waves through the wood sample, the diagonal terms of the elastic stiffness tensors were estimated.

\section{Results and discussion}

As only two specimens of each kind of sample were tested, no reliable statistical analysis could be performed. Thus, only half the measurement range (HMR) values (as a rough approximation of the standard deviation) are reported together with an estimate of the mean value in Figure 5 and Tables 1 and 2.

\section{Recent oak}

In the recent oak sample, the mean value of the longitudinal elastic modulus $\mathrm{E}_{\mathrm{L}}$ obtained by RUS was $0.6 \mathrm{GPa}$ higher $(+4 \%)$ than the static one for an HMR of $0.4 \mathrm{GPa}$ and $0.6 \mathrm{GPa}$, respectively (Figure $5 \mathrm{a}$ and Table 1). There are no significant differences between static and RUS measurement data. The same is true for the transverse elastic modulus $\mathrm{E}_{\mathrm{T}}$ with an almost similar trend but slightly greater differences. For the radial elastic modulus $E_{R}$, the mean RUS value is $0.7 \mathrm{GPa}$ higher $(+26 \%)$ than the static one for half range measurements of 0.58 and $0.15 \mathrm{GPa}$. This difference between RUS and compression test could be significant, but the apparently less accurate RUS results must be confirmed by additional measurements. The slightly greater differences in the $\mathrm{R}$ and $\mathrm{T}$ mean values than in the $\mathrm{L}$ direction mean values can probably also be explained by the growth ring curvature and greater sample misalignment in the RT plane. This hypothesis must be assessed in a future work.

No general trend can be observed in the shear moduli (Figure 5b and Table 1) between the RUS and static mean values, with a difference of up to $+0.28 \mathrm{GPa}(+29 \%)$ for $\mathrm{G}_{\mathrm{LT}}$ compared to an HMR close to $0 \mathrm{GPa}$ for RUS and around $0.3 \mathrm{GPa}$ for the compression test. HMRs are high (up to $45 \%$ of the mean value for $G_{\mathrm{LT}}$ ) for the compression test compared to the RUS test (up to $6 \%$ of the mean value for $\mathrm{G}_{\mathrm{LR}}$ ). This difference can be due to weaknesses in the static shear testing technique, where a manual identification of the pure shear area on the sample surface is required. This area is very limited in volume compared to that of the whole sample, and its width can be close to the ring width (Figure 1d). Some scatter can also be induced by the heterogeneity, which can be considerable for hardwood in the $\mathrm{R}$ and $\mathrm{T}$ directions (Hepworth et al. 2002). The area for strain characterisation in a static test can thus be affected by the variation in structural morphology, e.g. number of annual rings, ring curvature, and alignment of wood fibres, which lead to scatter in the estimated elastic properties. Microcracks in the LT plane were observed during the static shear test on one sample, and this can explain the particularly high HMR for $\mathrm{G}_{\mathrm{LT}}$ in the static test and the greatest difference in mean value from those obtained by RUS and US transmission. The microcracks probably did not influence the results of compression tests because the loading tends to close those that are perpendicular to the crack plane for $E_{R}$ measurement in the present case. In contrast, the first measured modes in RUS are particularly sensitive to the shear moduli that are then more reliably identified (Schubert et al. 2006; Longo et al. 2014). This explains the lower HMRs in that case and the good agreement with the US transmission values. It also appears that RUS measurements are not or are only slightly sensitive to microcracks considering the very low half range value for $G_{\text {IT }}$ This can be explained by the very small displacement that occurs during the sample vibration.

Apart from these discrepancies, the mean elastic moduli were not significantly different, compared to the HMRs, between the static, RUS, and US measurements. It can be safely concluded that all the moduli of the recent oak are "statistically" equivalent despite the difference in loading frequencies. This statement is in agreement with that of Gonçalves et al. (2011). Comparison with literature 

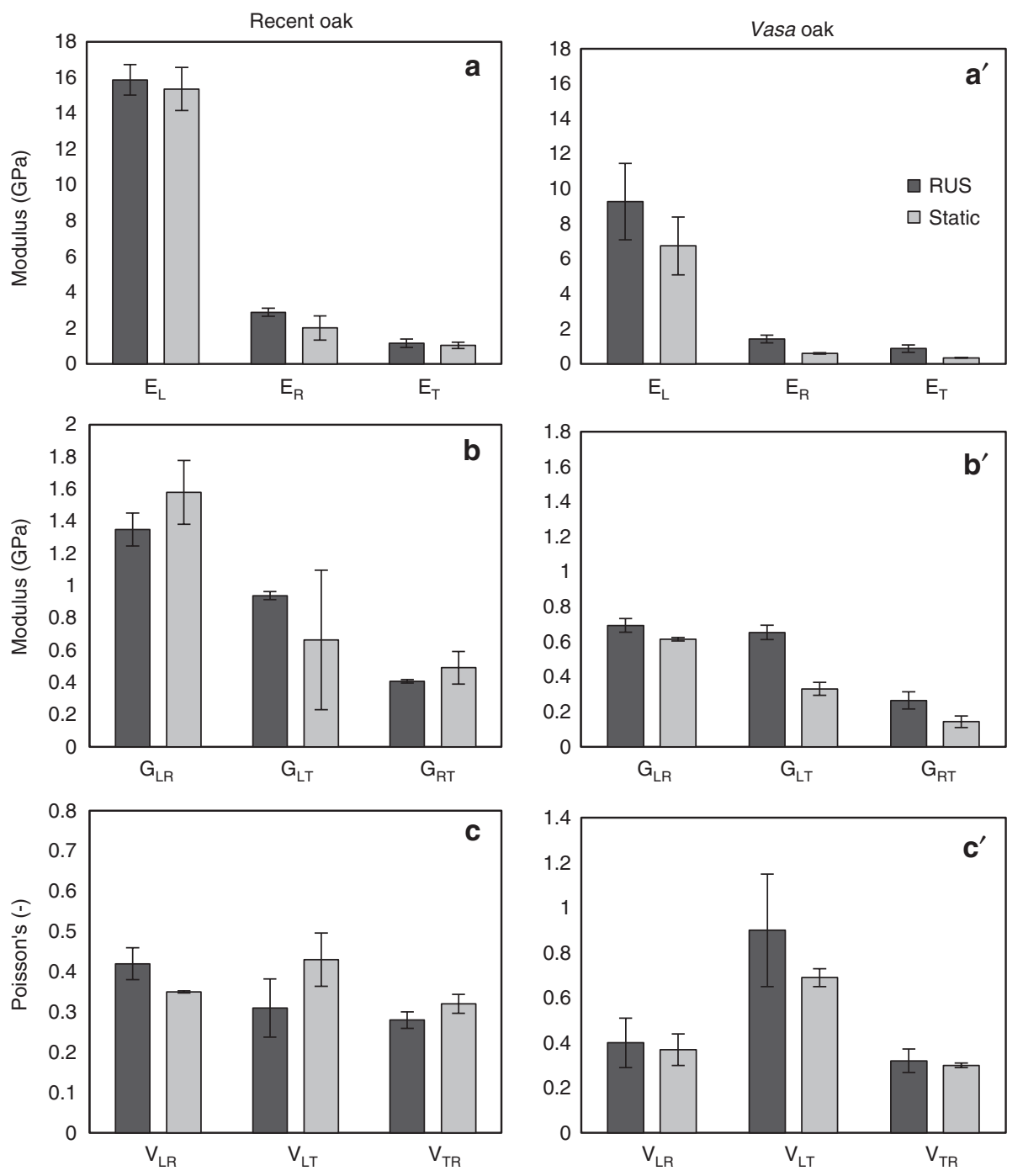

Figure 5: (a) Comparison of Young's moduli between RUS and static compression measurement for recent oak and (a') Vasa oak specimens. (b) Comparison of shear moduli from RUS and static shear measurements for recent oak and (b') Vasa oak specimens. (c) Comparison of Poisson's ratios from RUS and static measurements for recent oak and ( $\left.c^{\prime}\right)$ Vasa oak specimens.

data, compiled in Table 1, shows a general agreement if differences in density are taken into account. For example, $\mathrm{E}_{\mathrm{L}}$ is roughly proportional to $\rho$ (Guitard and El Amri 1987), and $\mathrm{E}_{\mathrm{L}} / \rho \sim 23.10^{6} \mathrm{~m}^{2} \mathrm{~s}^{-2}$ for de Borst et al. (2012) and Volkmer et al. (2014), whereas $E_{\mathrm{L}} / \rho \sim 22.10^{6} \mathrm{~m}^{2} \mathrm{~s}^{-2}$ in the present case for both static and RUS values.

The Poisson's ratios are compared in Figure $5 \mathrm{c}$ and Table 1. No general trend can be observed, and there is no obvious effect of the loading frequency on the Poisson's ratio. However, RUS measurements generally yield higher HMRs than the static test. Values obtained through static experiments are more reliable, as strain fields obtained for the whole area of the sample have been used. The relatively large scatter of the values determined by RUS can be explained by the very low sensitivity of the resonance frequencies to the Poisson's ratios (Longo et al. 2014). The best agreement with literature data was obtained with the present static values, but there is a substantial difference between each reference. This shows that measuring these coefficients is still challenging and needs further development. Note that with the sample containing microcracks in the LT plane, it was not possible to measure the corresponding Poisson's ratio $v_{\mathrm{LT}}$ during the compression test (only one value is available in Table 1).

\section{Vasa oak}

For the Vasa oak samples, the mean RUS value for $\mathrm{E}_{\mathrm{L}}$ was 3.9 GPa higher (+37\%) than the static one for HMRs of 1.4 

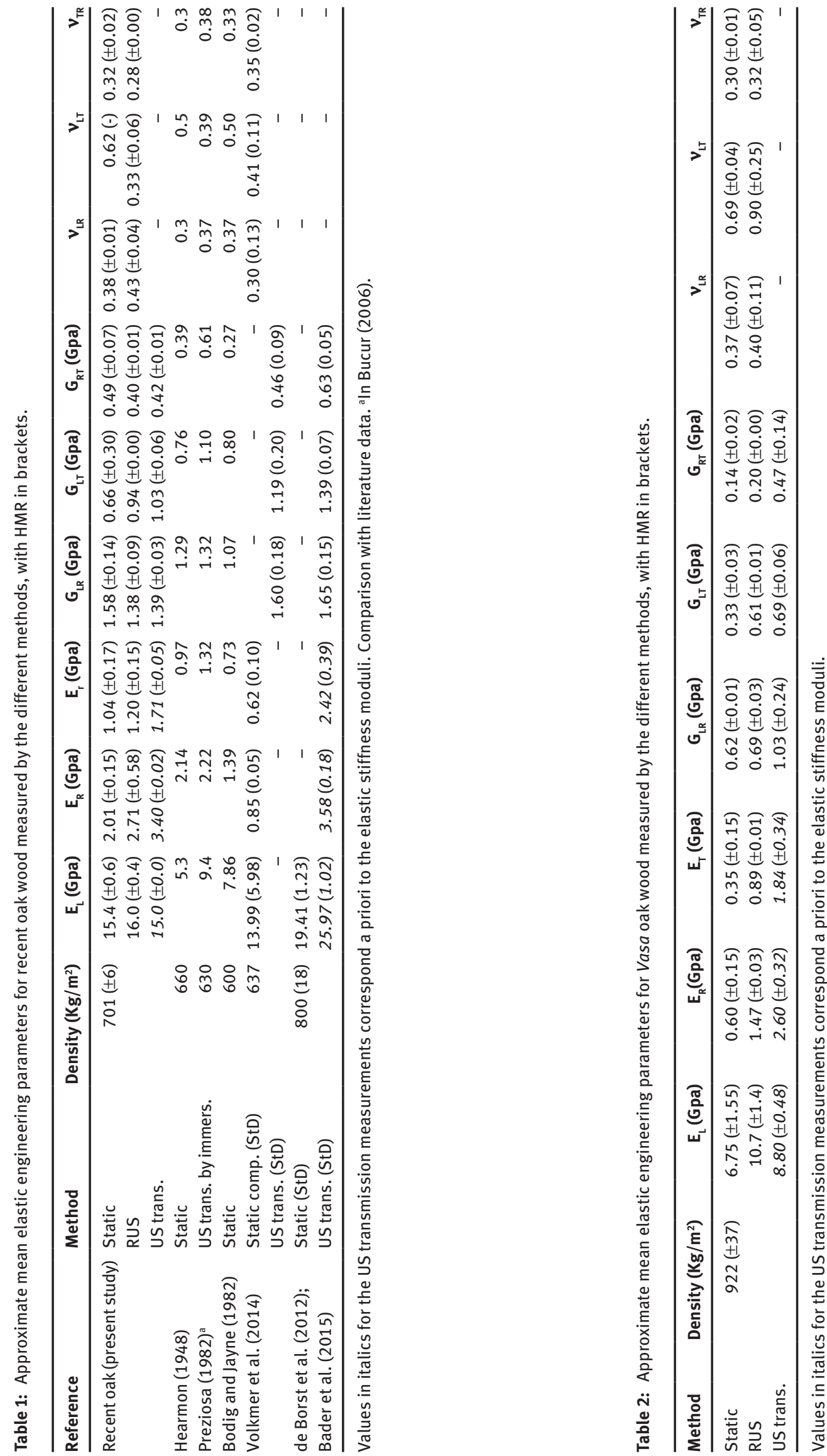
and $1.55 \mathrm{GPa}$ (Figure 5a' and Table 2). The high but similar measurement ranges for the RUS and compression tests were due to within-sample variability, e.g. differences in density (see the HMR for this parameter in Table 2) probably due to differences in PEG content, damage (Bjurhager et al. 2010), or microstructural characteristics of the cell wall (Bader et al. 2015). A significant difference between measurements obtained by the RUS and compression tests is observed as the difference in mean values was more than twice the HMR. This is particularly true for $E_{R}$ and $\mathrm{E}_{\mathrm{T}}$ as the differences in the mean values were $+0.87 \mathrm{GPa}$ $(+59 \%)$ and $+0.54 \mathrm{GPa}(+60 \%)$, respectively, for about 0.02 and $0.15 \mathrm{GPa}$ for the HMR of the RUS and compression tests, respectively. These differences in Young's moduli are presumably due to the viscous effect of PEG impregnation, in addition to the strong reduction in elastic moduli, especially in the transverse ( $\mathrm{R}$ and $\mathrm{T}$ ) directions, as reported by Bjurhager et al. (2012). Vasa oak clearly shows a more viscous behavior than the recent oak, with a more pronounced stress-strain hysteresis response during the static test (Figure 2) and a lower quality factor, i.e. the width of the resonance peak divided by the resonance frequency, for each resonance frequency peak of the RUS spectra (Figure 3).

A clear general trend was also observed in the case of the shear moduli (Figure $5 b^{\prime}$ and Table 2), where the HMRs are considerably smaller than those of the recent oak samples, especially in the case of the static test. The reason for this reduction is not clear and needs clarification. The differences between the mean values obtained between RUS and the static test, from $+0.08 \mathrm{GPa}(+11 \%)$ for $\mathrm{G}_{\mathrm{LR}}$ to $+0.28 \mathrm{GPa}(+46 \%)$ for $\mathrm{G}_{\mathrm{LT}}$, were again significantly larger than the HMR, e.g. around $0.02 \mathrm{GPa}$ for both methods. This increase in the shear moduli with increasing loading frequency is probably due to the viscous effect of the PEG impregnation, and its variable effect on each shear modulus still awaits an explanation. This sensitivity to the loading frequency is logically confirmed by the US transmission measurements that yield higher values than the RUS values. Moreover, the shear moduli, and especially $\mathrm{G}_{\mathrm{RT}}$, of the Vasa oak wood are on average drastically decreased as much as the Young's moduli by the PEG plasticisation.

The Poisson's ratios are compared in Figure $5 c^{\prime}$ and Table 2. As in the case of the recent oak samples, no general trend was observed, and there was no obvious effect of the loading frequency on the Poisson's ratio. Again, the values obtained through static experiments are more reliable than the RUS results (Longo et al. 2014). An interesting observation is that the Poisson's ratios for Vasa oak are almost the same as those of the recent oak, which suggests that PEG impregnation has a negligible effect on the Poisson's ratios.

\section{Conclusions}

RUS and static mechanical experiments have both advantages and drawbacks. In the case of the RUS technique, the speed at which the experiment is performed is the main advantage together with the easy and robust identification of the shear moduli. The small amount of material required can also be a benefit in the case of archaeological material. The RUS tests can be performed in different environmental conditions and do not require spacious installations, because the measuring device is relatively small. However, identification of all the elastic constants can be very challenging, especially in the case of the Poisson's ratios. On the other hand, classical static material testing requires more steps during the experiments, such as the installation and changing of setups for different experiments. Moreover, it is also difficult to achieve a pure compressive or pure shear loading in a representative volume of the specimen during static testing. However, the results reflect the actual quasi-static elastic parameters (RUS delivers dynamic parameters) more realistically under real loading conditions. Thus, the two techniques complement each other. A beneficial combination of both methods would allow accurate characterisation of all elastic constants of a single wood sample. By combining these two tests, the full elastic tensor and both dynamic and static elastic properties can be obtained on a single sample. In the present study, shear moduli and Poisson's ratios were determined for the Vasa oak, and the dependence of the data on the loading frequency was estimated.

Acknowledgments: This article was produced in collaboration with the National Maritime Museums and, in particular, the Vasa Museum. The work has been carried out within the "Support Vasa" project. Financial support for short-term scientific missions from EU COST Action FP1101 (Assessment, Reinforcement and modelling of Timber structures) is gratefully acknowledged.

\section{References}

Bader, T.K., Eberhardsteiner, J., de Borst, K. (2015) Shear stiffness and its relation to the microstructure of 10 European and tropical hardwood species. Wood Mat. Sci. Eng.: 1-10. Doi: $10.1080 / 17480272.2015 .1030773$. 
Bjurhager, I., Halonen, H., Lindfors, E.-L., Iversen, T., Almkvist, G., Gamstedt, E.K., Berglund, L.A. (2012) State of degradation in archeological oak from the 17th century Vasa ship: substantial strength loss correlates with reduction in (holo)cellulose molecular weight. Biomacromolecules 13:2521-2527.

Bjurhager, I., Ljungdahl, J., Wallström, L., Gamstedt, E.K., Berglund, L.A. (2010) Towards improved understanding of PEGimpregnated waterlogged archaeological wood: a model study on recent oak. Holzforschung 64:243-250.

Bodig, J., Jayne, B. Mechanics of Wood and Wood Composites. Van Nostrand Reinhold, New York, 1982.

Bucur, V. Acoustics of Wood. Springer Series in Wood Science. Springer, Berlin, Heidelberg, 2006.

Cederlund, C.O., Hocker, F. (2006) Vasa I: the archaeology of a Swedish royal ship of 1628 . In: Statens Maritima Museer. National Maritime Museum of Sweden.

Dahl, K.B., Malo, K.A. (2009) Planar strain measurements on wood specimens. Exp. Mech. 49:575-586.

de Borst, K., Bader, T.K., Wikete, C. (2012) Microstructure stiffness relationships of ten European and tropical hardwood species. J. Struct. Biol. 177:532-542.

Gonçalves, R., Trinca, A.J., Cerri, D.G.P. (2011) Comparison of elastic constants of wood determined by ultrasonic wave propagation and static compression testing. Wood Fiber Sci. 43:64-75.

Gonçalves, R., Trinca, A.J., Pellis, B.P. (2014) Elastic constants of wood determined by ultrasound using three geometries of specimens. Wood Sci. Technol. 48:269-287.

Guitard, D., El Amri, F. (1987) Modèles prévisionnels de comportement élastique tridimensionnel pour les bois feuillus et les bois résineux. Ann. Sci. Forest. 44:335-358.

Hassel, I., Berard, P., Moden, C.S., Berglund, L.A. (2009) The single cube apparatus for shear testing - Full-field strain data and finite element analysis of wood in transverse shear. Compos. Sci. Technol. 69:877-882.

Hearmon, R.F.S. Elasticity of wood and plywood. Report No. 7. HM Stationary Office, London, 1948.

Hepworth, D.G., Vincent, I.F., Stringer, G., Jeronimidis, G. (2002) Variations in the morphology of wood structure can explain why hardwood species of similar density have very different resistance to impact and compressive loading. Philos. T. R. Soc. A 360:255-272.

Huang, Y. H., Liu, L., Sham, F.C., Chan, Y.S., Ng, S.P. (2010) Optical strain gauge vs. traditional strain gauges for concrete elasticity modulus determination. Optik 121:1635-1641.

Kollmann, F. (1968) Mechanics and rheology of wood. In: Principle of Wood Science and Technology - 1. Solid Wood. Eds. Kollmann, F.P., Côté, W.A. Springer-Verlag, New York. pp. 292-419.

Ljungdahl, J., Berglund, L. (2007) Transverse mechanical behaviour and moisture absorption of waterlogged archaeological wood from the Vasa ship. Holzforschung 61:279-284.

Longo, R., Delaunay, T., Laux, D., El Mouridi, M., Arnould, O., Le Ciezio, E. (2012) Wood elastic characterization from a single sample by resonant ultrasound spectroscopy. Ultrasonics 52:971-974.

Longo, R., Laux, D., Pagano, S., Delaunay, T., Le Clezio, E., Arnould, O., (2014) Caractérisation élastique de bois de différentes densités par spectroscopie ultrasonore résonante (RUS). In: Matériaux 2014 - Conférence pluridisciplinaire sur les matériaux, 24-28 novembre 2014, Montpellier, France.

Majano-Majano, A., Fernandez-Kabo, J.L., Hoheisel, S., Klein, M. (2012) A test method for characterizing clear wood. Exp. Mech. 52:1079-1096.

Migliori, A., Darling, T.W., Baiardo, J.P., Freibert, F. (2001) Resonant ultrasound spectroscopy (RUS). In: Experimental Methods in the Physical Sciences. Eds. Levy, M., Bass, H.E., Stern, R. Elsevier. Vol. 39, pp. 189-220.

Ozyhar, T., Hering, S., Niemz, P. (2013) Moisture-dependent orthotropic tension-compression asymmetry of wood. Holzforschung 67:395-404.

Rakotovololonalimanana, H., Chaix, G., Brancheriau, L., Ramamonjisoa, L., Ramananantoandro, T., Thevenon, M.F. (2015) A novel method to correct for wood MOE ultrasonics and NIRS measurements on increment cores in Liquidambar styraciflua L. Ann. For. Sci. 1-9. 10.1007/s13595-015-0469-6.

Schubert, S.I., Gsell, D., Dual, J., Motavalli, M., Niemz, P. (2006) Rolling shear modulus and damping factor of spruce and decayed spruce estimated by modal analysis. Holzforschung 60:78-84.

Smith, I., Landis, E., Gong, M. Fracture and fatigue in wood. John Wiley and Sons, New York, 2003.

Sjödahl, M., Synnergren, P. (1999) A stereoscopic digital speckle photography system for 3-D displacement field measurements. Opt. Laser Eng. 31:425-443.

Toftegaard, H. (1999) Simulated stiffness determination from simple compression tests on a thick laminate. Compos. Part A-Appl. S. 30:849-858.

Tsoumis, G. Science and technology of wood. Structure, properties, utilization. Van Nostrand Reinhold, 1991.

Vázquez, C., Gonçalves, R., Bertoldo, C., Baño, V., Vega, A., Crespo, J., Guaita, M. (2015) Determination of the mechanical properties of Castanea sativa Mill. using ultrasonic wave propagation and comparison with static compression and bending methods. Wood Sci. Technol. 49:607-622.

Volkmer, T., Lorenz, T., Hass, P., Niemz, P. (2014) Influence of heat pressure steaming (HPS) on the mechanical and physical properties of common oak wood. Eur. J. Wood Prod. 72: 249-259.

Xavier, J., de Jesus, A.M.P., Morais, J.L.L., Pinto, J.M.T. (2012) Stereovision measurements on evaluating the modulus of elasticity of wood by compression tests parallel to the grain. Constr. Build. Mater. 26:207-215.

Zadler, B.J., Le Rousseau, J.H., Scales, J.A., Smith, M.L. (2004) Resonant ultrasound spectroscopy: theory and application. Geophys. J. Int. 156:154-169. 